\title{
Macrophages come to mind as keys to cognitive decline
}

\author{
D.G. Harrison' and Tomasz J. Guzik ${ }^{2,3}$ \\ 'Division of Clinical Pharmacology, Department of Medicine, Vanderbilt University Medical Center, Nashville, Tennessee, USA. ${ }^{2}$ Institute of Cardiovascular and Medical Sciences, University of Clasgow, \\ Glasgow, United Kingdom. ${ }^{3}$ Department of Medicine, Jagiellonian University College of Medicine, Krakow, Poland.
}

\begin{abstract}
Cognitive impairment, an underappreciated consequence of hypertension, is linked to cerebral arteriolar disease through poorly defined mechanisms. A study by Faraco et al. in this issue of the $J \mathrm{Cl}$ points to perturbations of neurovascular unit coupling caused by perivascular macrophages (PVMs) as a cause of hypertension-related cognitive impairment. Angiotensin II (Ang II) was shown to activate PVMs, causing them to produce superoxide and thereby alter the proper functioning of the adjacent arterioles. Faraco and colleagues also show that disruption of the blood-brain barrier occurs in hypertension, allowing circulating Ang II to access PVMs. This study provides important new insight into the role of inflammatory cells in the genesis of vascular dementia.
\end{abstract}

\section{Vascular dementia: a consequence of hypertension}

Stroke, renal failure, heart failure, and atherosclerosis are commonly recognized as consequences of hypertension; however, the association between hypertension and cognitive decline is not as widely appreciated. In the 1990s, Launer and colleagues reported that for every $10 \mathrm{mmHg}$ increase in midlife systolic pressure, there is a $5 \%$ increase in the incidence of cognitive decline, even after correction for stroke, coronary artery disease, and subclinical atherosclerosis (1). More recently, Ninoyama et al. reported that subjects with midlife hypertension had a five-fold increase in the risk of developing vascular dementia as compared with those without hypertension (2). To be fair, the association between hypertension and impaired cognition has not been uniformly reported, and other groups have reported a J-shaped curve, which in fact correlates better cognition with higher levels of blood pressure in very old individuals (3). A major problem with these studies is the lack of a precise definition of vascular dementia and difficulty in distinguishing this condition from Alzheimer's disease. Vascular dementia is tra- ditionally associated with stroke, sudden declines in cognition, or obvious perturbations of cerebral perfusion; however, these clinical clues are imprecise and often not apparent. Hypertension affects the small brain arterioles, leading to diffuse cerebral impairment, without localizing signs and with insidious progression, thereby making a clear distinction from Alzheimer's disease difficult. To complicate matters, in more than half of all cases of dementia, the pathology is mixed, with vascular disease coexisting with amyloid deposits (reviewed in ref. 4). Recently, accumulation of amyloid- $\beta$ has been observed in brains of hypertensive animals (5), and these amyloid deposits are reduced in mice lacking the angiotensin II type 1 receptor (6). Fascinatingly, $\beta$-amyloid has recently been shown to activate $\alpha-1$ adrenergic receptors by binding the first extracellular loop and thereby causing vasoconstriction (7). Thus, there is substantial interplay between the pathology of Alzheimer's disease and vascular dementia.

A fundamental aspect of hypertension is the profound vascular alterations that occur. Hypertension causes remodeling, fibrosis, and alterations of vascular tone

\section{Related Article: p. 4674}

that promote vasoconstriction, largely due to impaired endothelial function. Changes in vascular tone are often mediated by overproduction of ROS within the vessel, which directly promotes vasoconstriction and leads to oxidative inactivation of endothelium-derived nitric oxide. More than twenty years ago, it was recognized that angiotensin II (Ang II) is a potent stimulus for ROS production in vascular cells via activation of the NADPH oxidase (8). It is now recognized that Ang II can activate the NADPH oxidase in many cells and that this underlies many of the biological actions of Ang II, including vasoconstriction, sodium retention by the kidney, and sympathetic outflow from the brain (9). Indeed, scavenging of superoxide, or deletion of components of the NADPH oxidase, markedly blunts the hypertensive response to Ang II. Blockade of the angiotensin system also inhibits NADPH oxidase activity and improves endothelium-dependent vasodilatation in patients with hypertension. In the brain, endothelial signaling is critical for the function of the neurovascular unit (NVU), which allows coupling of nutrient delivery to neurons. As in other vessels, increased ROS formation plays a major role in disrupting NVU function and coupling (10).

There has been substantial interest in the cell type within the vessel wall responsible for ROS production, with a debate centered on whether intrinsic vascular cells, such as endothelial and vascular smooth muscle cells, or infiltrating inflammatory cells are the main ROS producers (11). In recent years, it has become increasingly evident that innate and adaptive immunity contributes to hypertension and the attendant end-organ damage. In peripheral vessels, monocytes, macrophages, and $\mathrm{T}$ cells invade the perivascular fat and adventitia and release mediators that promote vascular remodeling, fibrosis, and dysfunction (12-14). The adventitia is also home to resident macrophages with an $\mathrm{M} 2$ reparative phenotype. Professional phagocytes like these are sources of NADPH oxidase- 


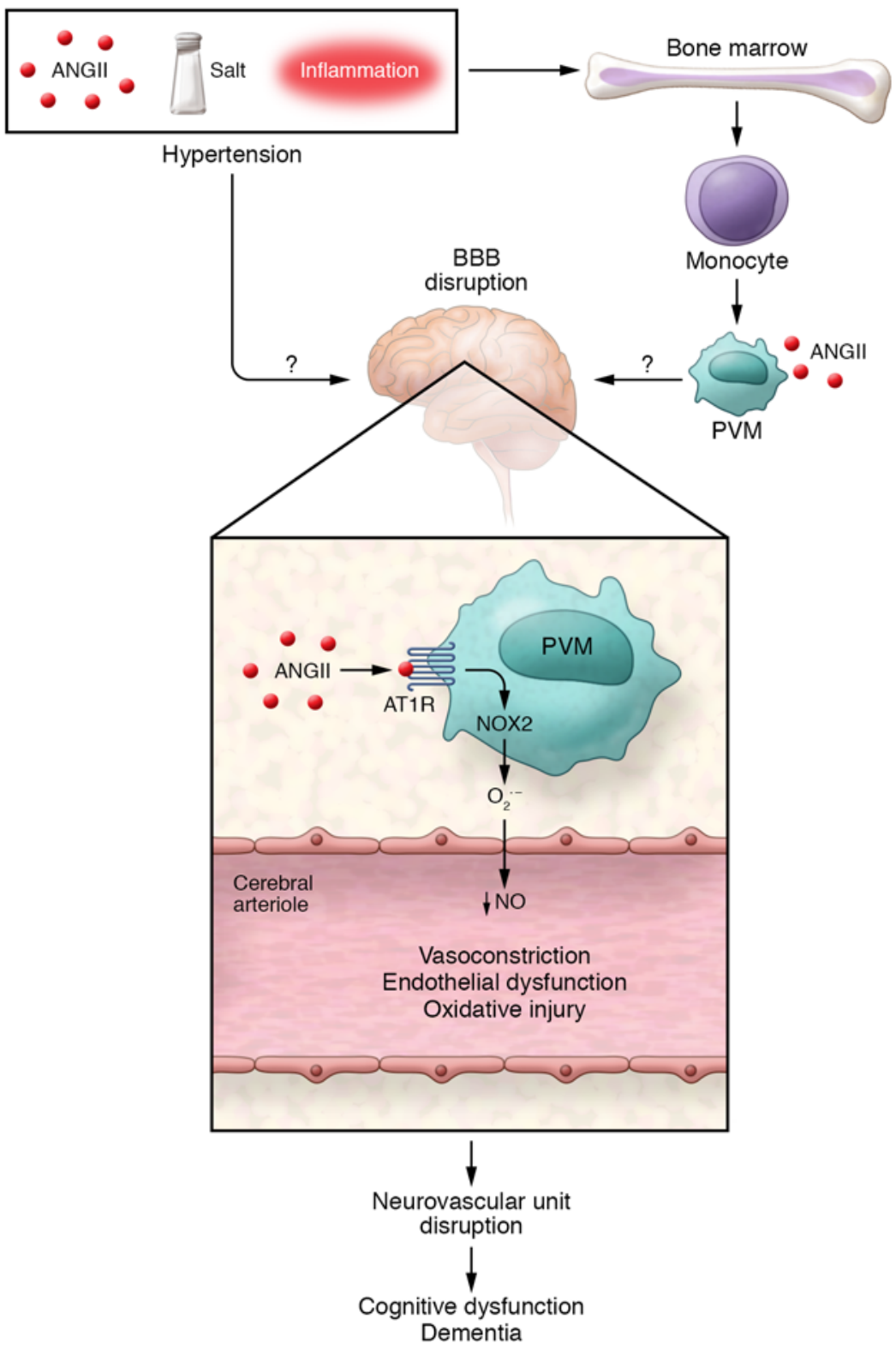

derived ROS, and, therefore, even a few such cells could contribute to oxidative signaling and injury if activated.

\section{Perivascular macrophages: the missing link}

In this issue, Faraco et al. provide an exciting link between hypertension and cognitive decline (15). Specifically, they have shown that perivascular macrophages (PVMs) closely associate with cortical arterioles and that hypertension causes disruption of the blood-brain barrier, allowing Ang II to reach these cells. Depletion of $\mathrm{PVMs}$ via intracerebroventricular injection of clodronate in two models of hypertension - a chronic fourteen-day Ang II infu-
Figure 1. Proposed role of PVMs in cognitive dysfunction. Hypertension leads to disruption of the blood-brain barrier, allowing Ang II and probably other neurohumoral agents to stimulate the production of ROS, including superoxide $\left(\mathrm{O}_{2}{ }^{-}\right)$, by PVMs. Superoxide and other ROS promote vasoconstriction, endothelial dysfunction, and oxidative injury in cerebral arterioles. These events cause dysfunction of the NVU, leading to cognitive impairment. AT1R, AT1 receptor; BBB, blood-brain barrier.

effects were confined to the local function of cerebral arterioles and their NVUs and were therefore not systemic. These interactions of PVMs with the NVU are illustrated in Figure 1.

\section{Future questions}

Faraco et al. carefully defined PVMs by surface marker expression and showed that this cell population is different from microglial cells. While Faraco and colleagues referred to PVMs as resident cells, their bone marrow transplantation studies clearly show that PVMs are derived from the bone marrow. Future studies to examine how PVMs are signaled to traffic from the marrow and how these cells find access to the cerebral arteriolar perivascular space will be very informative. PVMs express high levels of CD206, similar to M2 macrophages; however, determining the precise phenotype of these cells will require further analysis. Obviously, PVMs are capable of generating ROS but probably produce other mediators, such as cytokines and metalloproteinases that affect the vessel. PVMs also likely to respond to signals other than Ang II. A comprehensive transcriptomic analysis of cerebral cells like PVMs, similar to that being performed by the Functional Annotation of the Mammalian Genome (FANTOM) consortium, will provide further insight into the function and phenotype of PVMs (16).

Traditionally, the blood-brain barrier was thought to exclude hormones like Ang II from most of the brain, suggesting that any central signaling mediated by Ang II must be due to its actions on the circumventricular organs, which have a poorly formed blood brain-barrier, or due to Ang II produced locally within the brain. An important contribution of the study by Faraco et al. is the demonstration that circulating Ang II can cross the blood-brain barrier in hypertension and, thus, that Ang 
II not only affects cerebral perfusion, but likely signals other events, like increased sympathetic outflow from centers such as the paraventricular nucleus and the brain stem. Future studies using techniques such as those applied by Faraco et al. will be useful in defining the access of Ang II and other systemic mediators to various brain centers. Additional investigation into how the blood-brain barrier is disrupted in hypertension will also be of substantial interest.

Finally, the study by Faraco and colleagues (15) offers hints as to how we might use currently available therapies to reduce cognitive impairment. The results of this study suggest that AT1 receptor antagonists might be helpful in preventing cognitive impairment in hypertensive patients. Indeed, losartan has proven effective in ameliorating brain pathology and protecting brain function in a mouse model of Alzheimer's disease (17). Smaller clinical studies have suggested a benefit of AT1 receptor blockers in preventing cognitive impairment, although the results of larger trials have been disappointing (18). The length of treatment and timing of therapy may be important considerations. Newer agents, such as NOX inhibitors or scavengers of oxidative products, might also be effective (19). The effect of drugs like these on the PVM is now an important focus of future research.

\section{Acknowledgments}

This work was supported by grants from the American Heart Association (AHA) Strategically Focused Research Network (14SFRN20420046 and 14SFRN-
20770001); the NIH (R01HL039006, R01HL125865, 1P01HL129941, and P01GM015431); the Foundation for Polish Science Welcome (FNP/2009/Welcome02, to TJG); the Polish National Science Centre (Agreement 2011/03/B/NZ4/02454); and the International Senior Research Fellowship from the Wellcome Trust (to TJG).

Address correspondence to: David G. Harrison, 2220 Pierce Drive, Room 536 Robinson Research Building, Vanderbilt University, Nashville, Tennessee 37232, USA Phone: 615.875.3049; E-mail: david.g.harrison@vanderbilt.edu.

1. Launer LJ, Masaki K, Petrovitch H, Foley D, Havlik RJ. The association between midlife blood pressure levels and late-life cognitive function. The Honolulu-Asia Aging Study. JAMA 1995;274(23):1846-1851.

2. Ninomiya T, et al. Midlife and late-life blood pressure and dementia in Japanese elderly: the Hisayama study. Hypertension. 2011;58(1):22-28.

3. Richmond R, Law J, Kay-Lambkin F. Higher blood pressure associated with higher cognition and functionality among centenarians in Australia. Am J Hypertens. 2011;24(3):299-303.

4. Corriveau RA, et al. The Science of Vascular Contributions to Cognitive Impairment and Dementia (VCID): A Framework for Advancing Research Priorities in the Cerebrovascular Biology of Cognitive Decline. Cell Mol Neurobiol. 2016;36(2):281-288.

5 . Bueche CZ, et al. Hypertension drives parenchymal $\beta$-amyloid accumulation in the brain parenchyma. Ann Clin Transl Neurol. 2014;1(2):124-129.

6. Liu J, et al. Angiotensin type 1a receptor deficiency decreases amyloid $\beta$-protein generation and ameliorates brain amyloid pathology. Sci Rep. 2015;5:12059.

7. Haase $\mathrm{N}$, et al. Amyloid- $\beta$ peptides activate $\alpha 1$-adrenergic cardiovascular receptors. Hypertension. 2013;62(5):966-972.
8. Griendling KK, Minieri CA, Ollerenshaw JD, Alexander RW. Angiotensin II stimulates NADH and NADPH oxidase activity in cultured vascular smooth muscle cells. Circ Res. 1994;74(6):1141-1148.

9. Harrison DG, Gongora MC. Oxidative stress and hypertension. Med Clin North Am. 2009;93(3):621-635

10. Girouard H, Iadecola C. Neurovascular coupling in the normal brain and in hypertension, stroke, and Alzheimer disease. JAppl Physiol. 2006;100(1):328-335

11. Wang HD, et al. Superoxide anion from the adventitia of the rat thoracic aorta inactivates nitric oxide. Circ Res. 1998;82(7):810-818

12. Guzik TJ, et al. Role of the T cell in the genesis of angiotensin II induced hypertension and vascular dysfunction. JExp Med. 2007;204(10):2449-2460.

13. Madhur MS, et al. Interleukin 17 promotes angiotensin II-induced hypertension and vascular dysfunction. Hypertension. 2010;55(2):500-507.

14. Mikolajczyk TP, et al. Role of chemokine RANTES in the regulation of perivascular inflammation, T-cell accumulation, and vascular dysfunction in hypertension. FASEB J. 2016;30(5):1987-1999.

15. Faraco G, et al. Perivascular macrophages mediate the neurovascular and cognitive dysfunction associated with hypertension. JClin Invest. 2016;126(12):4674-4689.

16. Schultze JL, Freeman T, Hume DA, Latz E. A transcriptional perspective on human macrophage biology. Semin Immunol. 2015;27(1):44-50.

17. Ongali B, et al. Angiotensin II type 1 receptor blocker losartan prevents and rescues cerebrovascular, neuropathological and cognitive deficits in an Alzheimer's disease model. Neurobiol Dis. 2014;68:126-136.

18. Coca A. Hypertension and vascular dementia in the elderly: the potential role of antihypertensive agents. Curr Med Res Opin. 2013;29(9):1045-1054

19. Davies SS, et al. Treatment with a $\gamma$-ketoaldehyde scavenger prevents working memory deficits in hApoE4 mice. JAlzheimers Dis. 2011;27(1):49-59. 